\title{
PAPER \\ Visual Tracking in Occlusion Environments by Autonomous Switching of Targets
}

\author{
Jun-ichi IMAI ${ }^{\dagger a)}$ and Masahide KANEKO ${ }^{\dagger}$, Members
}

\begin{abstract}
SUMMARY Visual tracking is required by many vision applications such as human-computer interfaces and human-robot interactions. However, in daily living spaces where such applications are assumed to be used, stable tracking is often difficult because there are many objects which can cause the visual occlusion. While conventional tracking techniques can handle, to some extent, partial and short-term occlusion, they fail when presented with complete occlusion over long periods. They also cannot handle the case that an occluder such as a box and a bag contains and carries the tracking target inside itself, that is, the case that the target invisibly moves while being contained by the occluder. In this paper, to handle this occlusion problem, we propose a method for visual tracking by a particle filter, which switches tracking targets autonomously. In our method, if occlusion occurs during tracking, a model of the occluder is dynamically created and the tracking target is switched to this model. Thus, our method enables the tracker to indirectly track the "invisible target" by switching its target to the occluder effectively. Experimental results show the effectiveness of our method.

key words: visual tracking, occlusion, autonomous switching of targets, particle filter
\end{abstract}

\section{Introduction}

Visual tracking is one of the most important techniques in computer vision. For example, accurate real-time tracking is strongly required in human-robot interactions, because users with whom the robot interacts and objects to which the user and the robot share an interest are not necessarily static. Moreover, visual tracking techniques are also widely required by many vision applications such as video communications, surveillance systems and human-computer interfaces.

Such applications are assumed to be used in daily living spaces such as offices, and living rooms in homes. In these spaces, however, stable tracking is often difficult because there are many objects which can cause visual occlusion. Conventional tracking techniques especially cannot deal with complete occlusion by indefinite objects over long periods. They can handle only short-term or partial occlusion [1]-[4], or occlusion between known objects [5]. If complete occlusion is caused by some unknown object, the tracker in these techniques loses its target and only waits for the target to appear again in the view (or is trapped by a wrong target in the worst case). Furthermore, these techniques also cannot handle the case that an occluder such as

Manuscript received November 17, 2006.

Manuscript revised July 23, 2007.

${ }^{\dagger}$ The authors are with Graduate School of Electro-Communications, the University of Electro-Communications, Chofu-shi, 1828585 Japan.

a)E-mail: imai@ee.uec.ac.jp

DOI: 10.1093/ietisy/e91-d.1.86 a box and a bag contains and carries the target inside itself, that is, the case that the target invisibly moves while being contained by the occluder. In this case, even if the tracker memorizes the position where it loses the target, it cannot know that the target is not there anymore.

Then, how should the tracker cope with these situations? Our solution for this problem is as follows: "If the target is occluded and the tracker loses it, then the tracker should track its occluder instead of itself." We can indirectly estimate the position of the occluded target from the position of its occluder. Therefore, the tracker should pay an attention to an occluder when occlusion occurs.

In this paper, to cope with the occlusion problem, we propose a method for visual tracking by a particle filter, which switches tracking targets autonomously. In our method, if occlusion occurs during tracking, a model of the occluder is dynamically created and the tracking target is switched to this model. Disparity information obtained from a stereo camera is used for this dynamic creation of the occluder model. Since the original target model is also stored simultaneously, it can return to the tracking target if the occlusion ends and the original target appears again from behind the occluder. Thus, our method enables the tracker to indirectly track the "invisible target" by switching its target to the occluder effectively. Experimental results show the effectiveness of our method.

\section{Color-Based Particle Filtering}

\subsection{Particle Filter}

The particle filter, also known as the sequential Monte Carlo method, is an approach to Bayesian estimation of intractable posterior distributions from time series signals with nonGaussian noise [6], [7]. They are based on point mass representations of probability densities.

Let $s$ and $z$ denote the state of a tracking target and its observation respectively. The posterior distribution $p(\boldsymbol{s} \mid z)$ is approximated by a point mass set

$$
S=\left\{\left(s_{i}, \tilde{w}\left(s_{i}\right)\right) \mid i=1,2, \ldots, N\right\} .
$$

Each sample (called particle) $s_{i}$ represents a hypothetical state of the target with a corresponding weight $\tilde{w}\left(\boldsymbol{s}_{i}\right)$, where

$$
\sum_{i=1}^{N} \tilde{w}\left(s_{i}\right)=1 .
$$


Each particle transits to a new state according to a target dynamics model, and is weighted in terms of the observations. Then, $N$ particles are drawn with replacement, by choosing a particular particle $\boldsymbol{s}_{i}$ with a probability $\tilde{w}\left(s_{i}\right) \propto p\left(z \mid s_{i}\right)$, for the next time step. This process is called resampling.

Particle filtering handles multiple state hypotheses simultaneously and provides a robust tracking framework.

\subsection{Color Distribution Model}

In this paper, we adopt a color distribution as a target model, which is an improved version of that proposed by Nummiaro et al. [1]. This model has the following two advantages.

1. Robustness against non-rigidity, rotations and partial occlusions is achieved [1].

2. No prior knowledge is required to create a target model.

Especially the second advantage is suitable for our switching mechanism of targets, as discussed later.

Color distributions in this model are quantized into $C$ bins. Histograms are produced with a function $h(\boldsymbol{x})$, which assigns the color at a position $x$ in the image to the corresponding bin. In this paper, we use the HSV color space, but ignore the intensity values $(\mathrm{V})$ to make the tracker less sensitive to illumination conditions. The histograms are calculated in the HS plane using $C=16 \times 16=256$ bins.

We determine the color distribution inside an elliptic region $E$ with the position of its center $\left(c_{x}, c_{y}\right)$ and the length of half axes $a_{x}, a_{y}$. To increase the reliability of the color distribution when boundary pixels belong to the background or get occluded, smaller weights are assigned to the pixels which are further away from the region center by employing a weight function

$$
r(\boldsymbol{x} ; E)=1-\left(\frac{\left(x-c_{x}\right)^{2}}{a_{x}^{2}}+\frac{\left(y-c_{y}\right)^{2}}{a_{y}^{2}}\right),
$$

where $\boldsymbol{x}=(x, y)$ denotes the position of the pixel in the ellipse $E$. Then the color distribution $p(u ; E)(u=1,2, \ldots, C)$ in $E$ is calculated as

$$
p(u ; E)=\frac{\sum_{x \in E} r(x ; E) \cdot \delta(h(x)-u)}{\sum_{x \in E} r(x ; E)}
$$

where $\delta$ denotes the Kronecker delta function.

The state of each particle at time step $t$ is defined as an 8 -dimensional vector

$$
\begin{aligned}
& \boldsymbol{s}(t)=\left(c_{x}(t), c_{y}(t), a_{x}(t), a_{y}(t),\right. \\
& \left.c_{x}(t-1), c_{y}(t-1), a_{x}(t-1), a_{y}(t-1)\right)^{T},
\end{aligned}
$$

where $T$ denotes transposition. Since the elliptic region $E$ is unambiguously determined by the state $s$ (using the first four components), they are regarded as being the same in this paper $($ e.g. $p(\cdot ; E) \equiv p(\cdot ; s))$.

\subsection{Target Dynamics}

The state of each particle at the next time step is predicted by the target dynamics model

$$
\boldsymbol{s}(t)=A s(t-1)+v(t),
$$

where $A$ defines the deterministic component of the model and $v(t)$ is a multivariate random variable. In this paper, we use a first order model for $A$ describing a target dynamics with a constant velocity.

$$
A=\left(\begin{array}{cccccccc}
2 & 0 & 0 & 0 & -1 & 0 & 0 & 0 \\
0 & 2 & 0 & 0 & 0 & -1 & 0 & 0 \\
0 & 0 & 2 & 0 & 0 & 0 & -1 & 0 \\
0 & 0 & 0 & 2 & 0 & 0 & 0 & -1 \\
1 & 0 & 0 & 0 & 0 & 0 & 0 & 0 \\
0 & 1 & 0 & 0 & 0 & 0 & 0 & 0 \\
0 & 0 & 1 & 0 & 0 & 0 & 0 & 0 \\
0 & 0 & 0 & 1 & 0 & 0 & 0 & 0
\end{array}\right)
$$

Components of $v$ are independent and identically distributed, and each of them follows the Cauchy distribution

$$
p\left(v_{k}\right)=\frac{1}{\pi} \cdot \frac{\tau}{v_{k}^{2}+\tau^{2}} \quad(k=1,2, \ldots, 8),
$$

where $\tau$ is the scale parameter. The Cauchy distribution has a heavier tail than the Gaussian and is suitable to represent abrupt changes.

\subsection{Observation and Evaluation}

A distance measure $d_{B}$ between two color distributions $p$ and $q$ is defined as follows.

$$
\begin{aligned}
d_{B}(p, q) & =\sqrt{1-B(p, q)} \\
B(p, q) & =\sum_{u=1}^{C} \sqrt{p(u) q(u)}
\end{aligned}
$$

$B(p, q)$ is a discrete version of the Bhattacharyya coefficient [8], [9].

Each particle $s_{i}$ is evaluated by the following weight function $w$ based on the distance $d_{B}$ between the corresponding hypothetical color distribution $p\left(s_{i}\right)$ and the target model $q$.

$$
w\left(\boldsymbol{s}_{i}\right) \equiv w\left(p\left(\boldsymbol{s}_{i}\right)\right)=\exp \left(-\frac{d_{B}\left(p\left(\boldsymbol{s}_{i}\right), q\right)^{2}}{2 \sigma^{2}}\right)
$$

$w$ is specified by a Gaussian which is normalized to the range $[0,1]$ with variance $\sigma$. The re-normalized weight $\tilde{w}\left(s_{i}\right)$ is calculated as

$$
\tilde{w}\left(s_{i}\right)=\frac{w\left(s_{i}\right)}{\sum_{k=1}^{N} w\left(s_{k}\right)}
$$

for resampling.

Figure 1 shows an application of the color-based particle filter for tracking a stuffed bear as an example. In this 


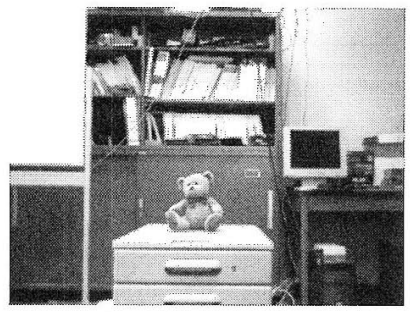

(a) Original image.

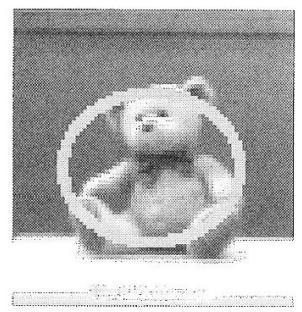

(b) Target.

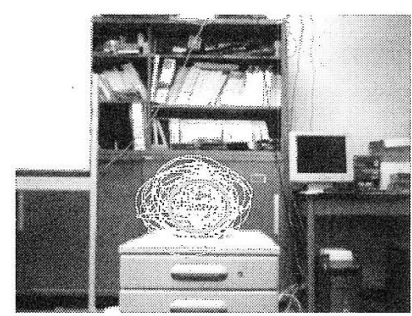

(c) Different hypotheses

Fig. 1 An example of the color-based particle filtering.

example, we set $\sigma=0.15$. Thick grey ellipse in Fig. 1 (b), (c) represents the expected position of the target, which is the region corresponding to the mean state $\overline{\boldsymbol{s}}$ calculated as

$$
\overline{\boldsymbol{s}}=\sum_{i=1}^{N} \tilde{w}\left(\boldsymbol{s}_{i}\right) \cdot \boldsymbol{s}_{i} .
$$

Thin white ellipses in Fig. 1 (c) represent the different hypotheses calculated by the particle filter.

\section{Autonomous Switching of Tracking Targets}

In this section, we propose an autonomous switching mechanism of tracking targets, which is used in the particle filter.

\subsection{Combination Model of Multiple Targets}

For realizing the autonomous switching of targets, the following functions are required of the tracker.

1. Detecting occlusion.

2. Creating and adding a new target model of an occluder dynamically if occlusion occurs.

3. Switching the tracking target back to the previous one if it appears again from behind the occluder.

4. Handling multiple occlusions, that is, the case that the occluder is then occluded by some other object.

To achieve these functions using the particle filter, we introduce a combination model of multiple targets

$$
w(s)=\sum_{j=0}^{M} \frac{w_{j}(s)}{\alpha^{j}},
$$

where $\alpha(>1)$ denotes a real constant. $w_{0}$ corresponds to the weight function for the target which should be originally tracked, and $w_{j}(j \geq 1)$ correspond to those for the occluders. $M$ denotes the number of the occluder models. The original target model is not counted in $M . M$ is set to 0 for the initialization and then $w=w_{0}$. Whenever occlusion occurs during tracking, a new model of the occluder is dynamically created and added to this combination model. This model also holds the original model and previously added models simultaneously, so it can handle multiple occlusions. Conversely, unnecessary models are eliminated whenever the corresponding occlusions end and the tracking target is switched back to the previous one.

In order to switch the tracking target back to the original one, the tracker must give the highest priority to the original target even after the tracking target has been switched to the occluder. Similarly, the target which is more previously added to the combination model must have the higher priority in the case of multiple occlusions. Our combination model realizes this mechanism by giving the $j$-th weight function $w_{j}$ an $\alpha$ times stronger influence on the whole weight than the $(j+1)$-th one $w_{j+1}$. As the simplest example, consider the case that $M=1$. In this case, the original target is occluded by only one occluder. Then the weight function is described as

$$
w(s)=w_{0}(s)+\frac{w_{1}(s)}{\alpha} .
$$

If only the occluder appears in the view, the first term in the weight function has little value (near 0 ) because the original target cannot be observed. Therefore, the second term is dominant in Eq. (15) and the occluder is tracked in this case. On the other hand, if the original target appears again and particles find it, the first term will have higher value (near 1 ) than the second one whose value is at most $1 / \alpha$. Therefore, the first term becomes dominant in Eq. (15) and the original target is tracked again. It is similar in the case that $M \geq 2$. Thus, the proposed combination model enables the tracker to switch the tracking target back to the previous one autonomously.

\subsection{Occlusion Detection}

As mentioned above, the occlusion detection is a necessary function for switching of targets. We decide whether occlusion occurs or not using a simple condition

$$
w(\overline{\boldsymbol{s}})<\frac{w_{T}}{\alpha^{M}},
$$

where $w_{T}$ denotes a predefined threshold. Equation (16) represents that the tracker lost sight of the current target during tracking for some reason. If this condition is satisfied, there are two possible situations.

1. The target is really occluded by some other object.

2. The tracker only temporally loses sight of the target for some reason such as an abrupt movement of the target and observation noise.

In the second case, it is expected that the tracking process returns to be normal within several time steps, and then switching of targets is not necessary. However, we cannot 
decide which is true at this point of time. So we formally decide that the occlusion has occurred if Eq. (16) continuously holds for predefined time steps $t_{T}$. Until then, the tracker continues the normal tracking process.

Preparing for the possibility of the first case, a model of a candidate for the occluder is created (as described in Sect. 3.3) at the point of time when Eq. (16) begins to hold. The created model, however, is not added to the combination model until the occlusion is formally identified (as described in Sect. 3.4). If the satisfaction of the condition breaks during $t_{T}$ steps, the tracker discards the created occluder model and returns to the normal tracking process.

Not only $w(\bar{s})$ but also the effective sample size defined as

$$
\hat{N}_{\text {eff }}=\frac{1}{\sum_{i=1}^{N}\left(\tilde{w}\left(s_{i}\right)\right)^{2}}
$$

is often used for evaluation of "goodness of tracking" in particle filtering [7]. $\hat{N}_{\text {eff }}$ is the approximate number of samples (particles) that actually contribute to estimate the mean state $\bar{s}$. However, what $\hat{N}_{\text {eff }}$ evaluates is the relative bias of normalized weights within all particles. (i.e. $\hat{N}_{\mathrm{eff}}=N$ if all particles have the same weight $\tilde{w}=1 / N$, and $\hat{N}_{\text {eff }}=1$ if $\tilde{w}=1$ for only one particle and $\tilde{w}=0$ for others.) Since the occlusion detection requires the absoloute evaluation of quality of tracking, it is considered that $\hat{N}_{\text {eff }}$ is not suitable for this purpose. So we adopt the criteria in Eq. (16) for the occlusion detection in this paper.

\subsection{Dynamic Creation of Occluder Model}

A color distribution model of a candidate for the occluder is dynamically created at the time step $t_{\text {occ }}$ when Eq. (16) begins to hold.

In this process, we integrate disparity information from a stereo camera with color information. The disparity is a quantity corresponds to a distance between the camera and objects in the view. A larger disparity means a shorter distance.

Let $\hat{d}(t)$ denote the mode of the disparity histogram in the elliptic region determined by the mean state $\bar{s}$ at time step $t . \hat{d}(t)$ is regarded as the representative disparity of the current target. Then the color distribution model of the candidate for the occluder is calculated as the following procedure (Fig. 2).

1. Calculate the disparity likelihood $p_{d}(\boldsymbol{x})$ based on $\hat{d}\left(t_{\mathrm{occ}}-1\right)$ (Fig. 2 (c)).

$$
p_{d}(\boldsymbol{x})= \begin{cases}1, & \epsilon>0 \\ 1-\epsilon^{2}, & -1<\epsilon \leq 0 \\ 0, & \epsilon \leq-1\end{cases}
$$

where

$$
\epsilon=\frac{d(x)-\hat{d}\left(t_{\mathrm{occ}}-1\right)}{d_{T}}
$$

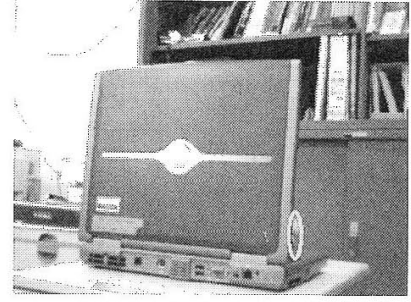

(a) Original image at $t_{n}$.

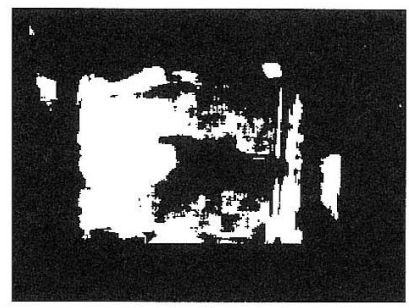

(c) Disparity likclihood map.

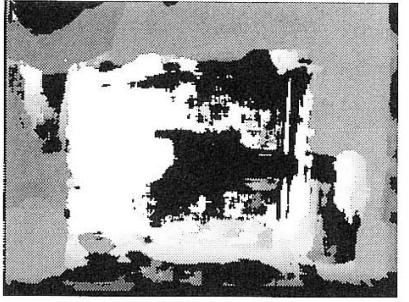

(b) Disparity image at $t_{o}$.

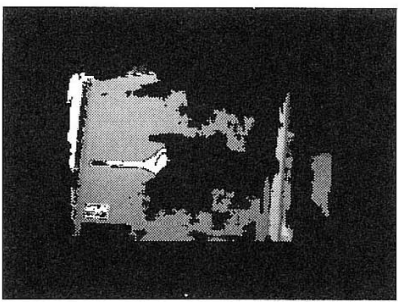

(d) Extracted region $R_{0}$.
Fig. 2 An example of the dynamic creation of the occluder model.

$d(x)$ denotes the disparity at the position $\boldsymbol{x}$ and $d_{T}$ denotes a predefined parameter. $p_{d}$ assigns 1 to pixels which have larger disparities than $\hat{d}\left(t_{\mathrm{occ}}-1\right)$, that is, those which exist nearer to the camera than the target at $t_{\mathrm{occ}}-1$. Preparing the possibility that the target moved further between $t_{\mathrm{occ}}-1$ and $t_{\mathrm{occ}}, p_{d}$ also gives some value to the pixel which has the same or only a little less disparity than $\hat{d}\left(t_{\mathrm{occ}}-1\right)$.

2. Calculate the color distribution $p_{\text {occ }}\left(u ; E_{\text {occ }}\right)$ of the tentative candidate for the occluder.

$$
p_{\mathrm{occ}}\left(u ; E_{\mathrm{occ}}\right)=\frac{\sum_{x \in E_{\mathrm{occ}}} p_{d}(\boldsymbol{x}) \cdot \delta(h(\boldsymbol{x})-u)}{\sum_{x \in E_{\mathrm{occ}}} p_{d}(\boldsymbol{x})}
$$

Equation (20) is the same as Eq. (4) except for the disparity likelihood instead of the weight concerning the ellipse region center (Eq. (3)). $E_{\text {occ }}$ is the elliptic region determined by the mean state $\bar{s}$ at $t_{\mathrm{occ}}$, except for too short length of the half axes of the ellipse. They are replaced with predefined values to avoid that the region size becomes too small.

3. Extract the candidate region $R_{\text {occ }}$ for the occluder (Fig. 2(d)).

$$
\begin{aligned}
R_{\mathrm{occ}} & =\left\{x \mid x \text { connected with } E_{\text {occ }} \cap \tilde{R}\right\} \\
\tilde{R} & =\left\{x \mid p_{d}(x) \cdot p_{\text {occ }}\left(h(x) ; E_{\text {occ }}\right)>0\right\}
\end{aligned}
$$

4. Calculate the color distribution $p_{\text {occ }}\left(u ; R_{\text {occ }}\right)$ of the final candidate for the occluder as Eq. (20).

Since this procedure is very simple and the disparity is often not so accurate, it is possible that the created model also does not necessarily have enough accuracy. The lack of accuracy is compensated by the online learning scheme described in Sect. 4.

\subsection{Combining Occluder Model}

If Eq. (16) continuously holds for $t_{T}$ steps, the created model 
of the occluder is added to the combination model. First, the weight function $w_{\text {occ }}$ for the occluder is defined by setting $q=p_{\text {occ }}\left(u ; R_{\text {occ }}\right)$ in Eq. (11). Then the number of occluder models $M$ is increased by 1 and $w_{M}$ is set to $w_{\text {occ }}$. Furthermore, the states of all particles are reset to $\overline{\boldsymbol{s}}\left(t_{\mathrm{occ}}\right)$ as the initial state for the new target.

\subsection{Elimination of Unnecessary Target Models}

As mentioned in Sect.3.1, the tracking target is autonomously switched back to the previous target model if the occlusion ends and the previous target appears again in the view. Then some of occluder models become unnecessary, so they should be eliminated.

We decide that the occlusion has ended if the following two conditions are satisfied.

1. The number of occluder models $M \geq 1$.

2. The weight for the mean state $\bar{s}$ given by the $M$-th weight function is not the maximum value of all $w_{j}(\overline{\boldsymbol{s}})$. That is,

$$
\max _{j} w_{j}(\overline{\mathbf{s}}) \neq w_{M}(\overline{\mathbf{s}}) .
$$

The second condition represents that the tracking target is now switched from the $M$-th model to the $j_{\max }$-th one, where $j_{\max }=\arg \max _{j} w_{j}(\bar{s})$. Then unnecessary models $w_{j}\left(j_{\max }<\right.$ $j \leq M$ ) are eliminated and $M$ is reset to $j_{\max }$.

\section{Online Learning of Target Model}

The quality of tracking with the color-based model is influenced by many factors such as illumination conditions, visual angles and camera parameters. To overcome the resulting appearance changes, we update the target model in an online fashion.

In order to discard unfavorable observations for occlusion or observation noise, the model is updated only if the condition

$$
w(\overline{\boldsymbol{s}}) \geq \frac{w_{T}}{\alpha^{M}}
$$

is satisfied. This is the reverse inequality of Eq. (16).

We adopt two kinds of the "mean color distribution" for the online learning. One is the color distribution for the mean state $p(u ; \bar{s})$ and another is the mixture of color distributions for all particles in $S$ calculated as

$$
\bar{p}(u)=\sum_{i=1}^{N} \tilde{w}\left(s_{i}\right) \cdot p\left(u ; s_{i}\right) .
$$

As we can see from Fig. 1 (b), it is expected that $\bar{p}$ includes more color information around the target besides that of the target itself than $p(\bar{s})$. Since especially the color distribution model of the occluder is possible to be incomplete, it is considered that using $\bar{p}$ as well as $p(\overline{\boldsymbol{s}})$ is effective to collect more information on the target widely and to make its model better. The tracker might drift if the influence of color
1. Perform the tracking process of the basic color-based particle filter.

a. Select $N$ samples from the set $S(t-1)$ with probability given by $w$.

b. Propagate each particle to a new states according to the target dynamics model (Eq. (6)).

c. Observe the color distributions and evaluate the weight of each particle by Eq. (14).

d. Calculate the normalized weights by Eq. (12).

e. Estimate the mean state $\bar{s}$ of the set $S(t)$ (Eq. (13)).

2. Check the occlusion condition (Eq. (16)).

a. Create the occluder model dynamically if Eq. (16) begins to hold.

b. Add the created model to the combination model if Eq. (16) continuously holds for $t_{T}$ steps.

3. Eliminate unnecessary target models.

4. Learn the target model in the online fashion.

a. Calculate $p(\overline{\boldsymbol{s}})$ and $\bar{p}$.

b. Update the target model as Eq. (26).

Fig. 3 The procedure of one iteration step in the proposed method.

information around the target introduced by $\bar{p}$ becomes too strong in the target model. However, the proportion of color information around the target is generally small in $\bar{p}$ because weights for particles which include the outside of the target is small. Therefore, to make the influence of color information around the target stronger, it needs to be continuously added to the target model. However, then the color information of the target itself is also added to the model much more than those around the target. So the color information around the target does not have the influence strong enough to cause the drift.

The color distribution $q$ is updated according to the equation

$$
\begin{aligned}
q(u ; t)=(1-\eta) & \cdot q(u ; t-1) \\
& +\eta \cdot((1-\beta) \cdot p(u ; \overline{\boldsymbol{s}})+\beta \cdot \bar{p}(u))
\end{aligned}
$$

for each bin $u$, where $\eta$ denotes the learning coefficient which weights the contribution of the new observation, and $\beta$ denotes the mixture rate determined by two weights $w(p(\overline{\boldsymbol{s}}))$ and $w(\bar{p})$ as follows.

$$
\beta=\frac{w(\bar{p})}{w(p(\overline{\mathbf{s}}))+w(\bar{p})}
$$

Figure 3 summarizes the procedure of one iteration step in the proposed method described in Sect. 2, 3 and 4.

\section{Experiments}

We carried out two experiments to show the effectiveness of the proposed method.

\subsection{Experimental Settings}

We use a Digiclops camera manufactured by Point Grey Research, Inc. as the stereo camera in our experiments. The 
color and disparity images are sequentially obtained from the camera. The image size is $320 \times 240$ pixels. The frame rate of our system is about 10 fps.

We set the number of particles $N=100$, the scale parameter of the Cauchy distribution $\tau=3$, the variance of the weight functions $\sigma=0.15$, the parameter in the combination model $\alpha=10$ and the learning coefficient $\eta=0.05$. Furthermore, we set the threshold $w_{T}=\exp (-2) \approx 0.1353$, which corresponds to the weight for $d_{B}\left(p\left(s_{i}\right), q\right)=2 \sigma$ in Eq. (11). Other thresholds are set as $t_{T}=5$ and $d_{T}=3$. These parameters have been determined experimentally. We discuss the parameter settings in Sect. 6.2.

Thick grey ellipse in each resultant image represents the region for the mean state $\bar{s}$ as well as in Fig. 1 (b), (c).

\subsection{Experiment 1}

First, we carried out an experiment to verify that our switching mechanism of targets works effectively under single occlusion. In this experiment, the camera itself moves around objects and the occlusion is caused by the change of visual angles.

Figure 4 and 5 show snapshots of a result of tracking and transition of the weight for the mean state $w(\bar{s})$ for Experiment 1, respectively. In Fig. 5, the solid line corresponds to the mean of 10 trials of tracking by the proposed method for the identical input images shown in Fig. 4. The dotted lines represent the region within the mean \pm standard deviation (SD). Two horizontal broken lines correspond to $w_{T}$ and $w_{T} / \alpha$ respectively. Note that the $y$-axis is log scale.

The original target in this experiment is a stuffed bear behind a notebook PC as shown in Fig. 4 (Frame \#33). The camera moves to left and the bear gets partially occluded by the PC (Frame \#74). At this point of time, the tracker does not lose the target by virtue of the ability of the colorbased particle filter. We can see also from Fig. 5 that the

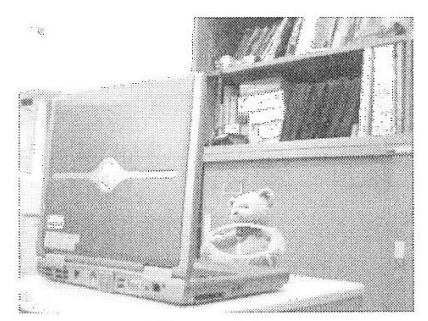

Frame \#33

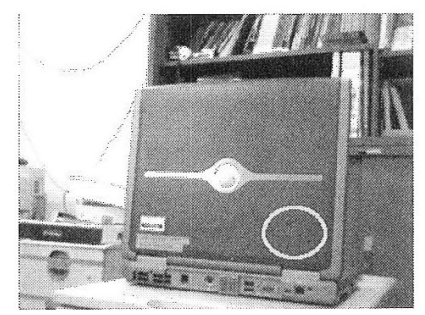

Frame \#93

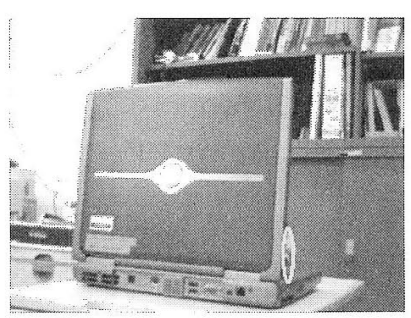

Frame \#74

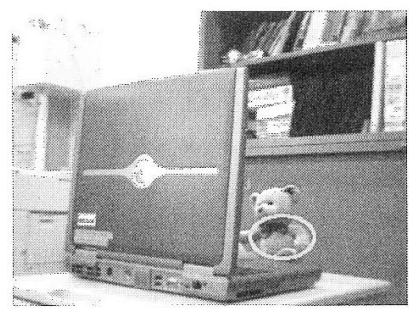

Frame \#155

Fig. 4 Snapshots of a result of tracking (Experiment 1). bear is still tracked because the weight $w(\overline{\boldsymbol{s}})$ remains over the threshold $w_{T}$. When the bear gets completely occluded, the tracking target is then switched to the PC (Frame \#93). Then the term $w_{1}(s) / \alpha$ for the PC is created and added to the weight function. Since this new term becomes dominant in the weight function, the range of $w(\bar{s})$ changes into $[0,0.1]$. Next, when the camera moves to right and the bear appears again from behind the PC, the tracking target is switched back to the bear correctly (Frame \#155). Then $w(\bar{s})$ recovers the value over $w_{T}$. These results show that the proposed method works effectively.

Figure 6 shows a comparison result between tracking only by the basic particle filter and by the proposed method. Thin white ellipses in Fig. 6 represent the different hypothetical regions as well as in Fig. 1 (c). In the case only by the basic particle filter (Fig. 6 (a)), when the bear gets completely occluded by the PC, particles lose sight of the target and spread out (Frame \#93), and then converge to the left down corner of the image wrongly (Frame \#155). Contrastively, the tracking target is switched to the PC as the occluder in the case by the proposed method (Fig. 6 (b)).

Of course, particles sometimes can find the bear and converge to it again even in the case only by the basic particle filter. However, the tracker in that case never know where the occluded target is. It just searches for its target in the view randomly.

\subsection{Experiment 2}

Next, we carried out an experiment for tracking under multiple occlusions and the situation that the target invisibly moves while being contained by the occluder.

Figure 7 and 8 shows snapshots of a result of tracking and transition of the weight for the mean state $w(\overline{\boldsymbol{s}})$ for Experiment 2, respectively. Note that the $y$-axis is $\log$ scale as well as Fig. 5. Three horizontal broken lines correspond to $w_{T}, w_{T} / \alpha$ and $w_{T} / \alpha^{2}$ respectively.

The original target in this experiment is a cushion held by a man as shown in Fig. 7 (Frame \#5). When the cushion is put into a cardboard box (Frame \#146), the tracker au-

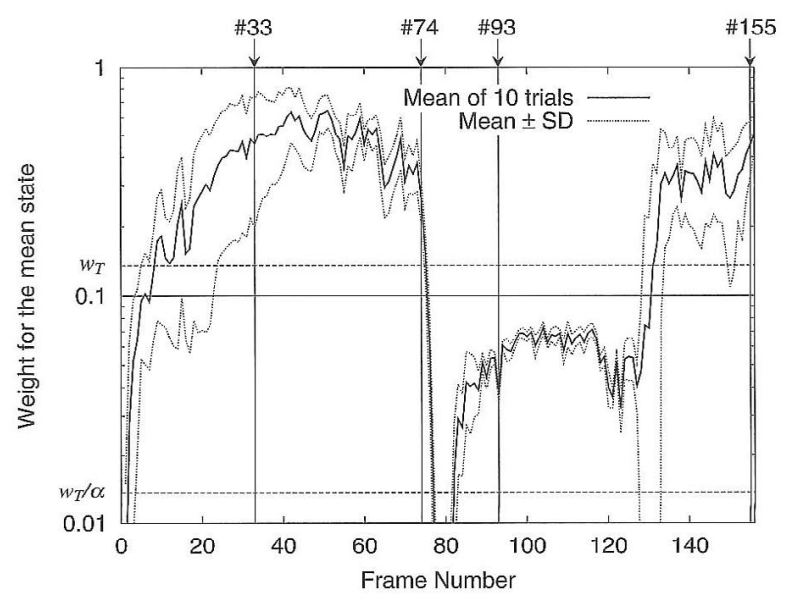

Fig.5 Transition of weight for mean state (Experiment 1). 

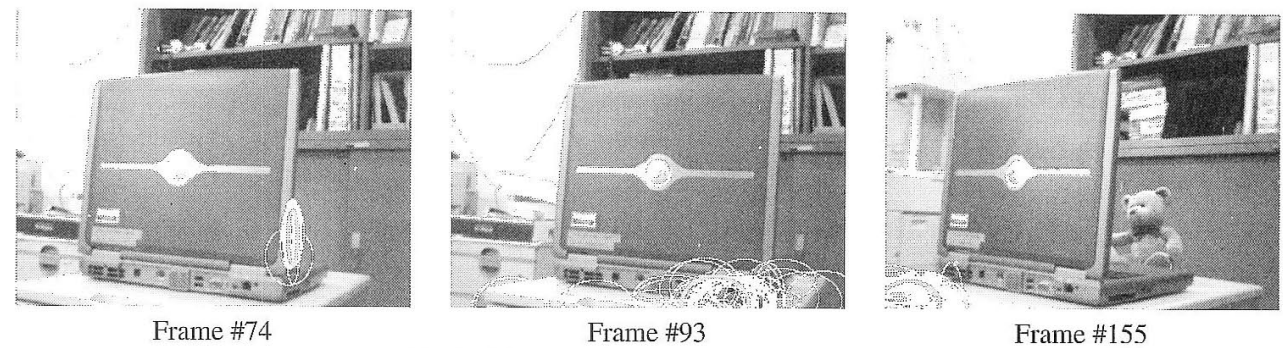

(a) Tracking only by the basic particle filter.

Frame \#155

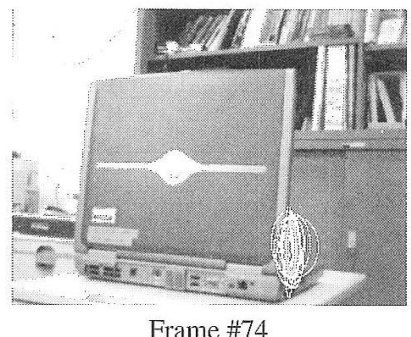

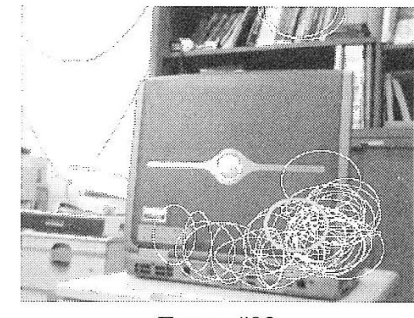

Frame \#93

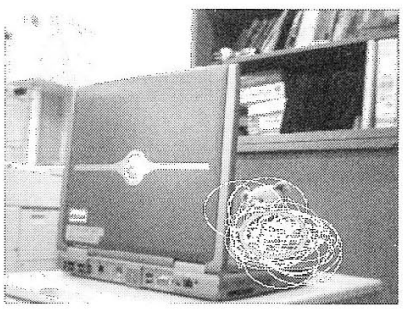

Frame \#155

(b) Tracking by the proposed method.

Fig. 6 A comparison result between the basic particle filter and the proposed method in Experiment 1.

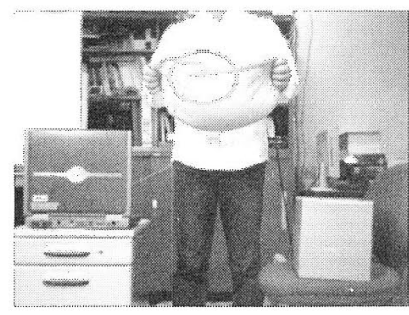

Frame \#5

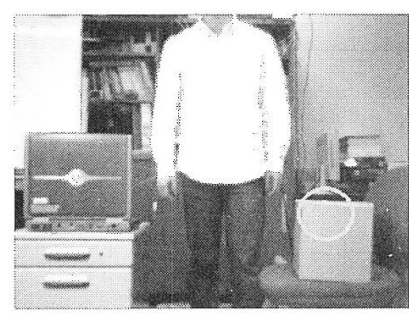

Frame \#205

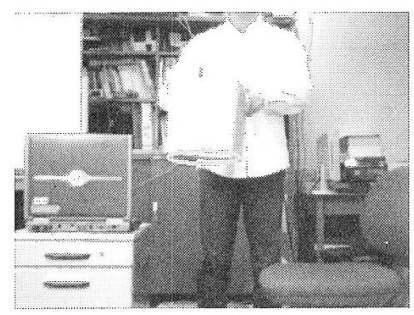

Frame \#300

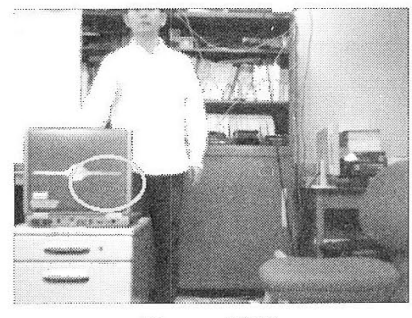

Frame \#423

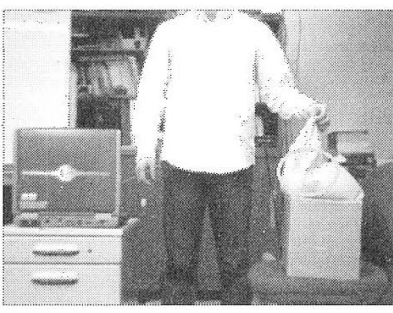

Frame \#146

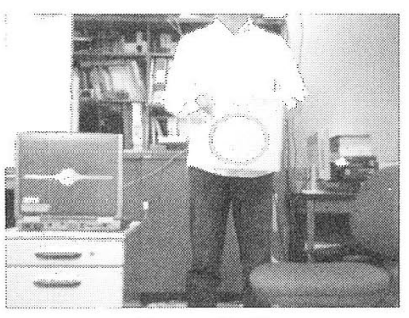

Frame \#285

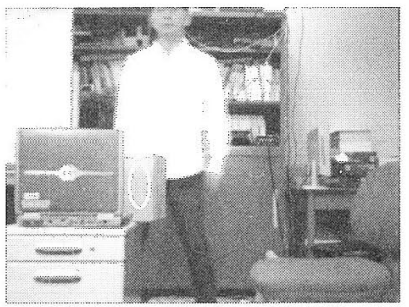

Frame \#391

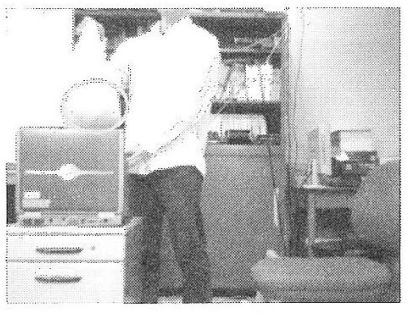

Frame \#562
Fig. 7 Snapshots of a result of tracking (Experiment 2).

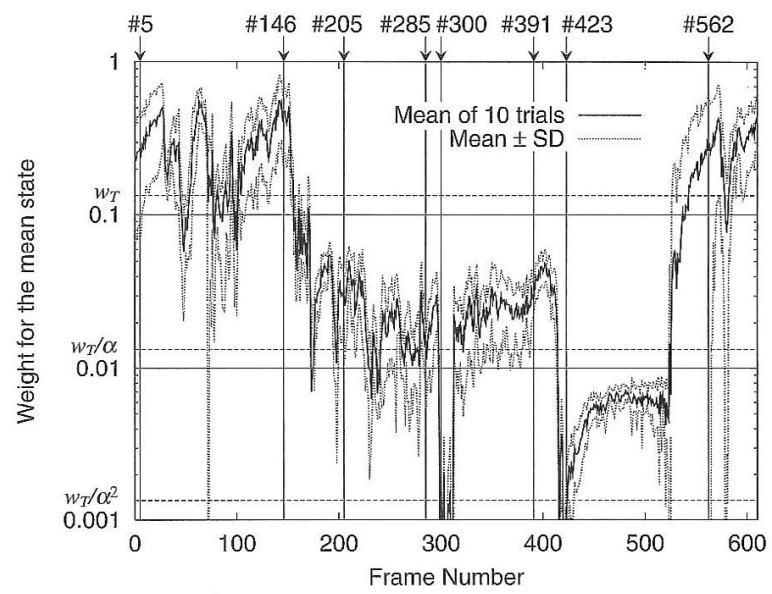

Fig.8 Transition of weight for mean state (Experiment 2).

tonomously switches its target from the cushion to the box (Frame \#205). Then the term $w_{1}(s) / \alpha$ for the box is added to the weight function and the range of $w(\bar{s})$ changes into $[0,0.1]$. The box is correctly tracked even if it is moved (Frame \#285). Then the cardboard box which contains the cushion is put behind a notebook PC (Frame \#391). When the box gets completely occluded, the tracker then autonomously switches its target to the PC (Frame \#423). Note that multiple occlusions occur and the term $w_{2}(s) / \alpha^{2} \in$ $[0,0.01]$ becomes dominant at this point of time. When the cushion is put out of the cardboard box behind the $\mathrm{PC}$, the tracking target correctly returns to the cushion as the original target (Frame \#562). If the box appeared again instead of the cushion at this time, the tracking target would be switched to the cardboard box.

While transition of the weight is somewhat unstable than that for Experiment 1, we can see from Fig. 8 that the proposed combination model and switching mechanism work effectively under the multiple occlusions. It is con- 
sidered that changes of illumination condition induced by changes of positions of the targets cause this instability, which the online learning scheme cannot absorb. Especially, a temporary fall of the weight appears near Frame \#300. (The tracker can catch only the edge of bottom of the box in Frame \#300.) At this point of time, while the tracker kept tracking the box, it wrongly switched its target to a dummy occluder which corresponds to the current target itself. So the term $w_{2}(s) / \alpha^{2}$ for the dummy was added and the range of $w(\overline{\boldsymbol{s}})$ changed into $[0,0.01]$ temporarily. Although the tracker discarded this dummy target soon in this experiment, it might keep tracking the dummy target wrongly in other situations. This problem should be improved to make the proposed method more robust.

\section{Discussions}

\subsection{Advantages and Limitations of Proposed Method}

As shown in the experimental results, the proposed method enables the tracker to indirectly track the "invisible target" by switching its target to the occluder appropriately. Position information of the occluder is greatly useful to estimate the position of the original target. Since conventional tracking techniques cannot handle the occlusion problem, this performance is a great advantage of the proposed method for surveillance systems and so on.

In future, we will aim to apply our method to the human-robot interactions especially. Our method enables an intelligent robot to pay an attention to the cardboard box or the PC while the robot and a user talk about the cushion, as shown in Fig. 7. That is, our method provides a way to handle multiple objects of interest simultaneously and to switch them appropriately. For the intelligent robot, deciding what it should watch is an important problem to obtain necessary information from its surroundings. Although a variety of attention models have been studied in the field of computer visions (e.g. [10]-[12]), our method which achieves autonomous switching of attention in occlusion environments is unique. It is expected that our method enables robots to perform more flexible and complicated interactions with users.

On the other hand, the limitation of the proposed method is the employment of a stereo camera. Although tracking process by particle filtering does not necessarily require a stereo camera, the dynamic creation of the occluder model requires the disparity information obtained from a stereo camera.

Furthermore, the proposed method is weak on situations that an occluded target moves behind a static occluder which occupies a large area in the image (e.g. a person walking behind a wall). If the occluder model is created accurately, the occluded target can be recaptured by particles around the occluder when it appears again even if it moves unpredictably behind the occluder. However, color and disparities of both ends of the large object often differ slightly in the image. Since the occluder model created dynamically tends to reflect the features of the end of the occluder where the target is occluded more strongly, the tracker often fails to catch the opposite end of the occluder. Then the tracker also often fails to recapture a person walking behind a wall. A solution of this problem is one of the important future works.

\subsection{Parameter Settings}

The constant $\alpha$ in the combination model (Eq. (14)) is the most important parameter in our method. $\alpha$ determines the degree of priority of the previous target. Since too small $\alpha$ may cause inappropriate switching of targets, it can be considered that the larger $\alpha$ is more desirable in this context. ( $\alpha$ must be greater than at least $1 / w_{T}$.) On the other hand, $\alpha$ also determines the upper bound of $M$ in Eq. (14). Because it is required to avoid that $w_{M}(\boldsymbol{s}) / \alpha^{M}$ and $w_{0}(\boldsymbol{s})$ have similar values for the same particle $s$ which tracks the $M$-th occluder correctly but cannot catch the original target at all.

In this paper, we set $\alpha=10$. Our preliminary experiment shows that the weight of the target model for the "background" is $10^{-5}$ or thereabouts. Therefore, it is expected that our system can cope with quadruple occlusions at least. It can be considered that this setting is practically reasonable.

Although details of settings of other parameters depend on user's system and problem to which the proposed method is applied, generally recommended values for the parameters are shown in Table 1. Numbers in parenthesis are values used in this paper. The followings are guidelines for determination of the parameters.

- The number of particles $N$ should be determined from the viewpoint of both accuracy of tracking and computational cost.

- The scale parameter of the Cauchy distribution $\tau$ determines the strength of randomness in the target dynamics model (Eq. (6)). Too small $\tau$ causes poor robustness against abrupt changes of target motion, and too large $\tau$ induces inaccurate prediction of target dynamics.

- The variance of the weight functions $\sigma$ affects the evaluation of particles. Since too small $\sigma$ makes the shape of weight functions too sharp, particles except those which can catch the target completely are given too small weights. Conversely, too large $\sigma$ causes inaccurate tracking because even particles of poor quality are given large weights.

Table 1 Recommended values for parameters.

\begin{tabular}{c|c}
\hline Parameter & Recommended values \\
\hline$N$ & $50-200(100)$ \\
$\tau$ & $1-5(3)$ \\
$\sigma$ & $0.10-0.20(0.15)$ \\
$\eta$ & $0.01-0.2(0.05)$ \\
$w_{T}$ & $0.1-0.2(0.1353)$ \\
$t_{T}$ & $3-5(5)$ \\
$d_{T}$ & $1-5(3)$ \\
$\alpha$ & $1 / w_{T}-100(10)$ \\
\hline
\end{tabular}


- The learning coefficient $\eta$ is a parameter for weighting the contribution of new observation in the online learning scheme. Similar approaches are often used for model updates in the field of image processing and $\eta$ is generally set to a small rate there.

- The threshold for the occlusion detection $w_{T}$ should be set to a small value.

- The threshold $t_{T}$ also should be set to a small value. However, too small $t_{T}$ will induce a confusion of temporally losing sight of the target with real occlusion.

- Determination of thresholds $d_{T}$ strongly depends on user's system and environment. It needs to be determined by preliminary experiments.

\subsection{Tracking by Color-Based Particle Filter}

Not only the particle filter but also the mean shift algorithm [13], [14] and the Kalman filter [15], [16] are often used for visual tracking. However, our tracking system is required to combine tracking of the current target with searching of the previous targets around the current one. Therefore, we need to adopt the particle filter which can simultaneously handle multiple hypotheses on the state of the target, instead of the methods which handle only one state hypothesis.

Furthermore, in tracking process, the system cannot know which object will become an occluder in advance. Since it is impractical that models of all objects in the surroundings are prepared in advance, the model of the occluder should be dynamically created according to need. The color distribution model has an advantage that no prior knowledge is required to create a new model as mentioned in Sect. 2.2. This is the reason why we adopt the color distribution as the target model in our system. On the other hand, it is also true that the color distribution model is not necessarily sufficient as the target model if it is applied to more complicated target objects and environments. A further study on the target model will be required to improve our system.

\subsection{Improvement of Proposed System}

In this paper, we have adopted a simple mechanism of switching targets for real-time processing. However, it is considered that there is still room for improvement in the proposed mechanism, especially in process of the dynamic creation of the occluder model described in Sect.3.3. For example, in the case that the target is held by a human, the tracker occasionally switches its target to his or her hand or arm wrongly instead of the correct occluder. To avoid the mistakes in switching like this, using other image features such as edges and textures as well as the color and disparity is expected to be effective. Furthermore, the online learning of the target dynamics [4] is also expected to be useful for predicting the position of the target and detecting a candidate for the occluder more accurately.
As mentioned in the previous section, the frame rate of our system is about 10 fps without any special optimization on a normal $3.2 \mathrm{GHz}$ PC. Our detail analysis shows that the most time-consuming process is obtaining the color and disparity images from the stereo camera. Since there is still room for optimization of this process in our system, an improvement in the frame rate can be expected. Furthermore, we also consider the employment of a special hardware for image processing.

\section{Conclusions}

In this paper, we have proposed a method for visual tracking by a particle filter, which switches tracking targets autonomously. In our method, the model of the occluder is dynamically created and the tracking target is switched to this model when occlusion occurs during tracking process. Thus, our method enables the tracker to indirectly track the "invisible target" under occlusion and the situation that the occluder contains and carries the target inside itself. Experimental results have shown the effectiveness of our method. Since conventional tracking techniques cannot handle the occlusion problem, it is expected that the performance of our method is greatly useful for many vision applications.

As a future work, the application of our method for the interactions between intelligent robots and users should be addressed. Furthermore, improvements of our method are also required to make it more robust and accurate as described in Sect. 6.

\section{References}

[1] K. Nummiaro, E. Koller-Meier, and L.V. Gool, "An adaptive colorbased particle filter," Image Vis. Comput., vol.21, no.1, pp.99-110, 2003.

[2] S. Zhou, R. Chellappa, and B. Moghaddam, "Visual tracking and recognition using appearance-adaptive models in particle filters," IEEE Trans. Image Process., vol:13, no.11, pp.1491-1506, 2004.

[3] H.T. Nguyen and A.W.M. Smeulders, "Fast occluded object tracking by a robust appearance filter," IEEE Trans. Pattern Anal. Mach. Intell., vol.26, no.8, pp.1099-1104, 2004.

[4] T. Bando, T. Shibata, K. Doya, and S. Ishii, "Switching particle filters for efficient visual tracking," Robotics and Autonomous Systems, vol.54, no.10, pp.873-884, 2006.

[5] T. Yang, S.Z. Li, Q. Pan, and J. Li, "Real-time multiple objects tracking with occlusion handling in dynamic scenes," Proc. IEEE Computer Society Conf. on Computer Vision and Pattern Recognition, vol.1, pp.970-975, 2005.

[6] M. Isard and A. Blake, "CONDENSATION - Conditional density propagation for visual tracking,” Int. J. Comput. Vis., vol.29, no.1, pp.5-28, 1998

[7] A. Doucet, N. de Freitas, and N. Gordon, eds., Sequential Monte Carlo Methods in Practice, Springer, New York, 2001.

[8] T. Kailath, "The divergence and Bhattacharyya distance measures in signal selection," IEEE Trans. Commun. Technol., vol.COM-15, no.1, pp.52-60, 1967.

[9] F. Aherne, N. Thacker, and P. Rockett, "The Bhattacharyya metric as an absolute similarity measure for frequency coded data," Kybernetika, vol.32, no.4, pp.1-7, 1997.

[10] L. Itti, C. Koch, and E. Niebur, "A model of saliency-based visual attention for rapid scene analysis," IEEE Trans. Pattern Anal. Mach. Intell., vol.20, no.11, pp.1254-1259, 1998. 
[11] Y.F. Ma and H.J. Zhang, "A model of motion attention for video skimming," Proc. IEEE Int. Conf. Image Processing, vol.1, pp.129$132,2002$.

[12] J.S. Cho and B.J. Yun, "Selective-attention correlation measure for precision video tracking," IEICE Trans. Inf. \& Syst., vol.E88-D, no.5, pp.1041-1049, May 2005.

[13] C. Yang, R. Duraiswami, and L. Davis, "Efficient mean-shift tracking via a new similarity measure," Proc. IEEE Computer Society Conf. Computer Vision and Pattern Recognition, vol.1, pp.176-183, 2005.

[14] M.H. Jeong, B.J. You, Y. Oh, S.R. Oh, and S.H. Han, "Adaptive mean-shift tracking with novel color model," Proc. IEEE Int. Conf. Mechatronics and Automation, vol.3, pp.1329-1333, 2005.

[15] F. Dellaert and C. Thorpe, "Robust car tracking using Kalman filtering and Bayesian templates," Proc. SPIE: Intelligent Transportation Systems, vol.3207, pp.72-83, 1997.

[16] D. Ponsa, A. Lopez, J. Serrat, F. Lumbreras, and T. Graf, "Multiple vehicle 3D tracking using an unscented Kalman filter," Proc. 8th Int. IEEE Conf. Intelligent Transportation Systems, pp.1108-1113, 2005 .

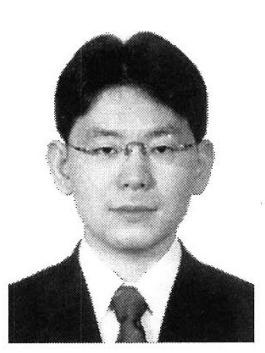

Jun-ichi Imai received his B.E., M.E., and Ph.D. degrees from Hokkaido University, Japan in 1997, 1999, and 2003, respectively. From April 2003 to April 2005, he was a postdoctoral fellow at Satellite Venture Business Laboratory, Muroran Institute of Technology. In May 2005, he joined the University of ElectroCommunications as a research associate. He is currently an assistant professor of Department of Electronic Engineering. His research interests are in intelligent systems and human-robot interactions. He is a member of Information Processing Society of Japan, Japan Society of Kansei Engineering, and the Institute of Image Information and Television Engineers.

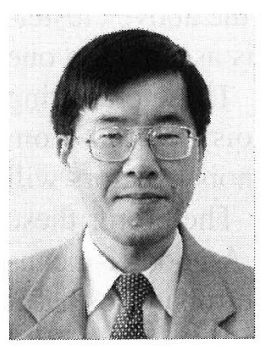

Masahide Kaneko received his B.E., M.E., and D.E. degrees from the University of Tokyo, Japan in 1976, 1978, and 1981, respectively. From April 1981 to March 1994, he was with the Research and Development Laboratories of Kokusai Denshin Denwa Co., Ltd. (KDD). From April 1994 to March 1997, he was an associate professor of Department of Information and Communication Engineering, the University of Tokyo. In April 1997, he was reinstated in the Research and Development Laboratories of KDD. In April 1998, he joined the University of ElectroCommunications as an associate professor. He is currently a professor of Department of Electronic Engineering. His research interests include the image coding, 3D image processing, processing of facial image information, and active interaction between humans and intelligent robots. He is a member of IEEE, the Institute of Image Information and Television Engineers, the Information Processing Society of Japan, the Robotics Society of Japan, and Japan Academy of Facial Studies. 